\title{
Correlation of epithelial-mesenchymal transition and MACC1 in squamous cell carcinoma of tongue
}

\author{
Gongfa Wu ${ }^{1}$, Chen Chun ${ }^{2}$, Qing Sun ${ }^{3}$, Yeqing Liu ${ }^{3}$, Jingjing Han ${ }^{1}$, Chaobin Pan ${ }^{4}$, Zhiquan Huang He $^{4,5}$ Haigang Li*1,3,5 $^{*}$ \\ ${ }^{1}$ Department of Pathology, People's Hospital of Zengcheng Distric, Guangzhou 511300, China \\ ${ }^{2}$ Department of Stomatology, People's Hospital of Zengcheng Distric, Guangzhou 511300, China \\ ${ }^{3}$ Department of Pathology, Sun Yat-sen Memorial Hospital, Sun Yat-sen University, Guangzhou 510120, China \\ ${ }^{4}$ Department of Oral and Maxillofacial Surgery, Sun Yat-sen Memorial Hospital, Sun Yat-sen University, Guangzhou 510120, China \\ ${ }^{5}$ Guangdong Provincial Key Laboratory of Malignant Tumor Epigenetics and Gene Regulation, Sun Yat-sen Memorial Hospital, Sun Yat-sen University, \\ Guangzhou 510120, China
}

\begin{abstract}
Objective: Eepithelial-mesenchymal transition (EMT) represents conversion of an epithelial cell in an elongated cell with mesenchymal phenotype. EMT plays an important role in nvasion and migration of tumor cells in malignant tumors, which is associated with poor prognosis, high metastatic rate, and low disease-free survival time. Metastasis-associated in colon cancer-1 (MACC1), which is identified as an oncogene to promote metastasis and proliferation, induces the EMT in gastric carcinoma. In the present study, we investigated EMT and its correlation to the expression of metastasis-associated in colon cancer 1 (MACC1) in squamous cell carcinoma of tongue (TSCC).
\end{abstract}

Method: The expression of E-cadherin, vimentin, and MACC1 was detected in 65 paraffin-embedded tissue specimens of TSCC, atypical hyperplasias (AH), and adjacent normal squamous epithelium (ANSE) tissues by using immunohistochemistry (IHC).

Results: Abnormal expression of E-cadherin, which included reduction in cytomembrane and increase in cytoplasm, was observed in both all AH and TSCC. Reduction E-cadherin expression in cytomembrane was associated with advanced tumor stage and less survival time in TSCC ( $\mathrm{P}=0.002$ and 0.000$)$. Increase expression of vimentin was observed and associated with lymphoid metastasis, advanced tumor stage and less survival time in TSCC ( $\mathrm{P}=0.025$, 0.033 , and , 0.000 respectively). The reduction E-cadherin expression in cytomembrane and increase expression of vimentin were associated with MACC1 overexpression (both $\mathrm{P}=0.000)$

Conclusions: Our data suggest that abnormal expression of E-cadherin and vimentin in tumor tissues, and the EMT process may occur and play an important role in the development of TSCC. Abnormal expression of E-cadherin includes reduction of membranous expression and increase of cytoplasmic expression, and the reduction of membranous expression may be more important. The abnormal expression of E-cadherin and vimentin is associated with MACC1 in TSCC.

\section{Introduction}

Oral squamous cell carcinoma (OSCC) is the most common cancer for head and neck squamous cell carcinoma, which is the sixth most common cancer worldwide. OSCC accounts for 2-3\% for all malignant tumors. Tongue squamous cell carcinoma (TSCC) is the most common carcinoma of OSCC, accounting for $25-40 \%$ of mouth neoplasms [1]. TSCC has an increasing incidence in recent years. It is absolutely a major public health problem. In spite of wide use of combination therapy, such as operation-radio-chemo-therapy, the mortality rate of TSCC is still not optimistic compared to the previous. The 5-year survival rate of TSCC is only 50-60\% [2]. An estimated 300,400 new cases and 145,400 deaths from oral cavity cancer occurred in 2012 worldwide [3].

Epithelial-mesenchymal transition (EMT) is a biological event in which epithelial cells lose many of their phenotypic features and gain extra properties typical of mesenchymal cells. Gaining mesenchymal signature facilitates detachment of tumor cells, accompanied by proteolytic digestion of basement membrane, vascular invasion, and migration of circulating tumor cells to distant sites [4]. The metastasisassociated in colon cancer1 (MACC1) gene has been identified by a genome-wide screen of human colon cancer, and is a prognostic biomarker for tumor progression, metastasis and survival of a variety of solid cancers including colorectal cancer [5]. We recently identified that MACC1 expression was highly associated with lymphatic metastasis and poor overall survival in patients with TSCC [6]. MACC1 promotes gastric cancer cell proliferation and invasion by inducing EMT through activation of the hepatocyte growth factor (HGF)/c-Met signaling pathway [7].

E-cadherin (Epitheia-cadhein) and vimentin are EMT marker proteins. E-cadherin is a calcium-dependent transmembrane glycoprotein located in the epithelial tissue, which is an important cell adhesion molecule and signal transduction factor, can direct the formation of protein complexes attached to the actin cytoskeleton in combination with beta-catenin formation which can prevent and

Correspondence to: Dr. Haigang Li. Department of Pathology, Sun Yat-sen Memorial Hospital, Sun Yat-sen University, Guangzhou 510120, China; E-mail: 13728089120@126.com

Key words: carcinoma, squamous cell carcinoma of tongue, E-cadherin, vimentin, MACC1

Received: July 11, 2016; Accepted: August 14, 2016; Published: August 16, 2016 
reduce tumor cell adhesion. During EMT, E-cadherin expression is reduced to near-zero level [4]. As a mesenchymal marker not expressed in normal epithelial cells, vimentin can be acquired during EMT [8].

The aim of our study was to investigate immunohistochemically whether MACC1 was associated with EMT in TSCC and whether EMT marker protein detection predicted the lymphoid metastasis and prognosis of TSCC 65 patients.

\section{Material and methods}

\section{Patients and tissue samples}

All evaluated samples of formalin-fixed, paraffin embedded from 65 patients of Chinese origin with TSCC for immunohistochemistry (IHC) were collected from the department of pathology in Sun Yat-sen Memorial Hospital from 1996 to 2006. The median age at the time of diagnosis was 51 years (range 25 to 77 ), including 13 patients $\leq 40$ years. None of the patients had received chemotherapy of radiation before surgery. The tumors were graded histologically as well-differentiated (12 patients, $18.5 \%$ ), moderately differentiated (50 patients, $76.9 \%$ ), and poorly differentiated ( 3 patients, $4.6 \%$ ). Of the 65 TSCC patients, local lymph node metastasis occurred in 17 cases (26.2\%). According to the WHO classification of TSCC, tumors were classified as stage I (15 patients, $23.1 \%$ ) II (27 patients, $41.5 \%$ ) III (9 patients, $13.8 \%$ ) and IV (14 patients, $21.5 \%)$.

\section{Immunohistochemistry}

65 paraffin-embedded specimens of TSCC, including 18 atypical hyperplasias $(\mathrm{AH})$ and 42 adjacent normal squamous epithelium (ANSE) tissues were examined imumnohistochemically. The staining was performed with the anti- E-cadherin (4A2C7, ready to use, Maxim, China), vimentin (SP20 ready to use, Maxim, China), MACC1 antibody (polyclonal, 1:100, Abcam ab106005) according to the standard protocols. Briefly, after being deparrffined, tissue sections were blocked sequentially with $3 \% \mathrm{H}_{2} \mathrm{O}_{2}$ and normal serum and then incubated with primary antibody at $4{ }^{\circ} \mathrm{C}$ overnight. Further, tissue sections were incubated with biotinylated secondary antibody and conjugated with streptavidin-HRP complex (ready to use SP kit, Zhongshan, China). Finally, sections were visualized with 3,3'-diaminobenzidine and counterstained with hematoxylin. Colon cancer tissues were used as positive control for MACC1, breast carcinomas for E-cadherin and vimentin. PBS was used instead of the primary antibodies for the negative controls.

\section{Evaluation of IHC staining}

The IHC evaluation of the tissues was conducted by 2 pathologists (G.W and H.L) who assessed the number of positive cells and the intensity of staining. The positive results were judged by semiquantitative points.

The expression of E-cadherin was classified as follows [9]: 1) "preserved" (positive): membranous immunostaining in more than $50 \%$ of the epithelial tumor cells; or 2) "reduced" (negative): membranous immunostaining in $50 \%$ or less of the epithelial tumor cells. We also used $50 \%$ as a threshold value to evaluate cytoplasmic immunostaining.

The expression of vimentin was classified as follows [10]: 1) "negative": cytoplasmic immunostaining in less than $10 \%$ of the epithelial tumor cells; or 2) "positive": cytoplasmic immunostaining in $10 \%$ or more of the epithelial tumor cells.
The staining intensity of MACC1 was scored 0 (negative), 1 (weak), 2 (medium) and 3 (strong). The integral of the rate of positive cells was $0(0 \%), 1(1-25 \%), 2(26-50 \%)$ and $3(>50 \%)$. The staining intensity score and the proportional score were added to obtain the total score. A total score $<3$ was considered to represent low MACC1 expression [11].

\section{Statistical analysis}

All of the statistical analyses were conducted using SPSS 13.0 statistical software. The Wilcoxon X, Mann-Whitney U-test, $t$ test and Kruskal-Wallis analyses were used to analyze the correlation between E-cadherin, vimentin expression and the clinicopathological characteristics. The relationship between E-cadherin, vimentin and MACC1 expression was assessed by the Pearson method. T test was used to compare the average survival time of different groups. The survival curves were plotted using the Kaplan-Meier method and were compared with the log-rank test. $\mathrm{P}<0.05$ was considered to indicate a statistically significant result.

\section{Results}

Expression of E-cadherin and vimentin is abnormal in TSCC compared with non-cancerous epithelial tissue adjacent to the carcinoma

In all the ANSE, E-cadherin strong positive expression was limited to the cell surface membrane. The basal layer presented membranous staining with occasional cytoplasmic staining. In the parabasal and intermediate cell layers, E-cadherin immunoreactivity was found circumferentially at the cell membrane, but the staining was lost at the superficial layer (Figure 1A). This gradually decreasing pattern of membranous staining in the normal tongue was not found in the $\mathrm{AH}$ and invasive TSCC. Reduction of E-cadherin expression in cytomembrane was observed in both all AH and TSCC (Figure 1A to 1D). Meanwhile, weak or strong E-cadherin expression was observed in cytoplasm in both of them. The staining pattern of weak or strong membranous immunoreactivity and increased cytoplasmic immunoreactivity could be found in TSCC whether the foci were invasive or not.

No vimentin expression was observed in ANSE and AH. 27 TSCC were counted as vimentin positive expression according to the evaluation of IHC staining (Figure 1E to $1 G$ ).
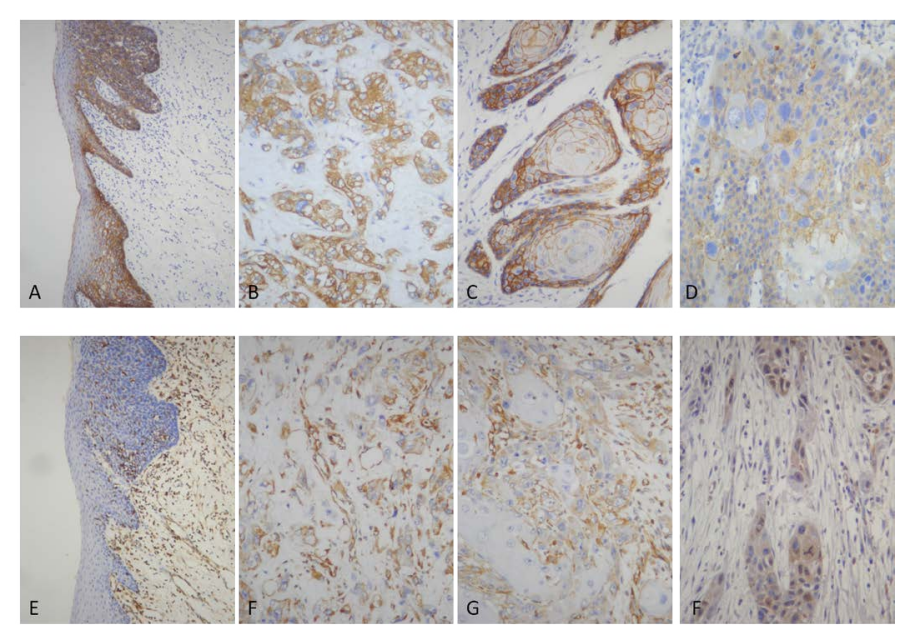

Figure 1: 


\section{Reduced membranous expression of E-cadherin is associated with advanced stage and less survival time in TSCC}

Because reduction of membranous expression and increase cytoplasmic expression of E-cadherin were present in all cases, we separately assessed the tumor cells as membranous expression or cytoplasmic expression and then we tried to correlate positive rate with the main clinocopathological parameters (tumor differentiation, lymphoid metastasis, TNM stage, and survival time).

37 of 65 TSCC were counted as membranous positive expression of E-cadherin according to the evaluation of IHC staining, 44 as cytoplasmic positive expression. The results of immunoreactivity and its association with clinicopathological parameters were summarized in Table 1. The E-cadherin membranous expression was associated with a lower TNM stage, I(13/15), II(16/27), III(3/9), IV(5/14) (P=.002). The survival time of E-cadherin membranous positive group (58.19 \pm 30.37) was longer significantly than that of the negative group (19.79 $\pm 18.27)(\mathrm{P}=0.000)$. Survival analysis showed the patients with low E-cadherin membranous expression levels demonstrated a significantly shorter survival time (Figure 2 and Table 1, $\mathrm{P}=0.000$ ). No association of E-cadherin membranous expression was found with gender, age, tumor differentiation and lymphoid metastasis $(\mathrm{P}=0.862,0.384,0.339$ and 0.130 , respectively). No association of E-cadherin cytoplasmic expression was found with gender, age, tumor differentiation, lymphoid metastasis, TNM stage, and survival time $(\mathrm{P}=0.497,0.599,0.406,0.768$, 0.409 , and 0.117 , respectively).

26 TSCC were counted as both membranous and cytoplasmic positive expression of E-cadherin according to the evaluation of IHC staining, 11 as only membranous positive expression, 18 as only cytoplasmic positive expression, and 10 as negative for membranous and cytoplasmic immunoreaction. No relationship was found between E-cadherin membranous and cytoplasmic immunoreactions $(\mathrm{P}=0.616)$.

Increase expression of vimentin is associated with lymphoid metastasis, advanced stage and less survival time in TSCC

The expression of vimentin was evaluated as positive in 27 cases of TSCC. The reactivity was obvious in tumor cells from both the centre of poor-differentiated tumor islands and the periphery of proliferative islands in the cutinized tumor. The vimentin expression was associated with lymphoid metastasis, in group with lymphoid metastasis (11/17), and the group without lymphoid metastasis $(16 / 48)(\mathrm{P}=0.025)$. The vimentin expression was associated with a higher TNM stage, $\mathrm{I}(4 / 15)$, II (9/27), III(6/9), IV(8/14) $(\mathrm{P}=0.033)$. The survival time of vimentin expression group was less significantly than that of the negative group $(t$ test, $\mathrm{P}=0.000$ ). Survival analysis showed the patients with high vimentin expression levels demonstrated a significantly shorter survival time (Figure 2 and Table 1, $\mathrm{P}=0.043$ ). No association of vimentin expression was found with gender, age, tumor differentiation $(\mathrm{P}=0.594,0.708$, 0.650 , respectively).

\section{Relationship between E-cadherin and vimentin expression in TSCC}

The vimentin positive expression was detected in 6 of 37 cases with E-cadherin membranous positive expression, Pearson's analysis revealed that the membranous expression of E-cadherin was negatively correlated with vimentin expression in TSCC $(\mathrm{r}=-0.591, \mathrm{P}=0.000)$. No association was found between the vimentin and E-cadherin cytoplasmic expression $(\mathrm{P}=0.699)$, as showed in Table 2 .

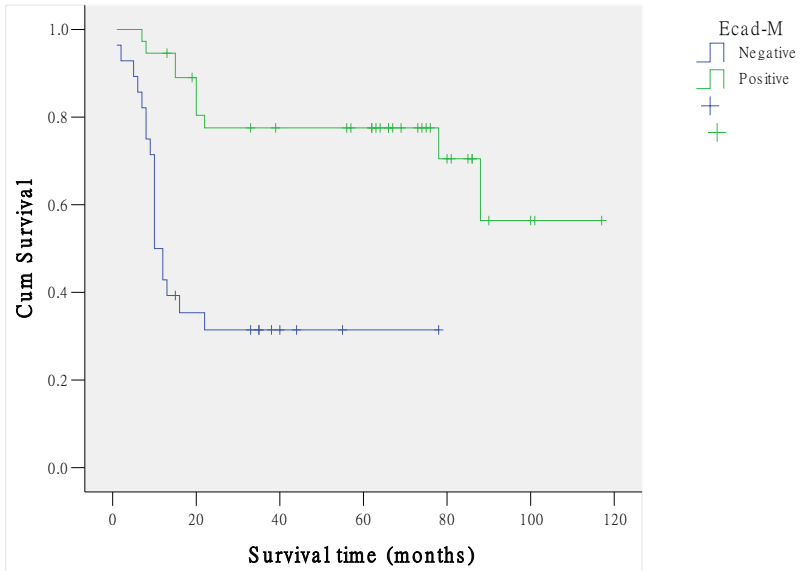

A

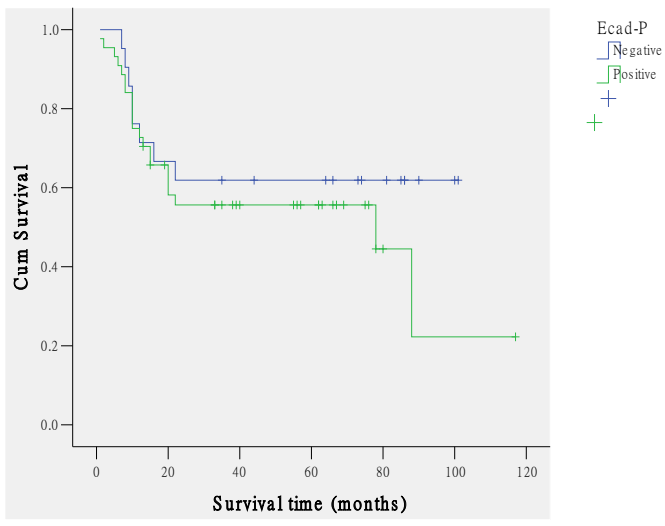

B

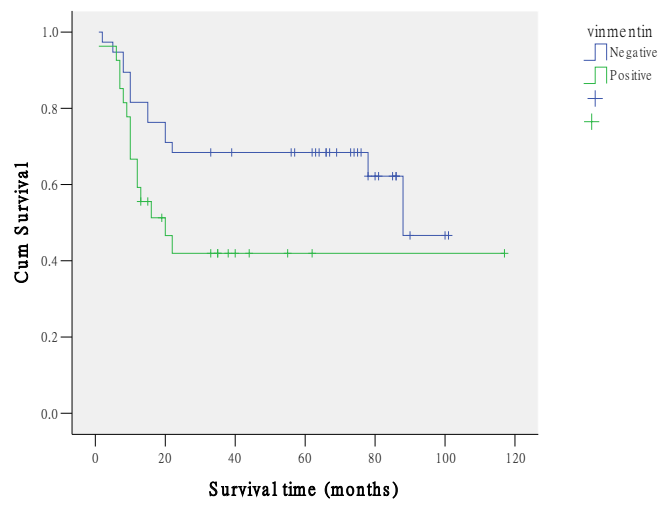

Figure 2:

Reduction membranous expression of E-cadherin and increase expression of vimentin are associated with MACC1 overexpression in TSCC

The MACC1 high expression was detected in 7 of 37 cases with E-cadherin membranous positive expression, Pearson's analysis revealed that the membranous expression of E-cadherin was negatively correlated with MACC1 expression in TSCC $(\mathrm{r}=-0.495, \mathrm{P}=0.000)$. High expression was detected in 18 of 27 cases with vimentin expression, Pearson's analysis revealed that the vimentin expressions was positively 
Table 1. Relation of E-cadherin with vimentin in TSCC.

\begin{tabular}{|c|c|c|c|c|c|c|}
\hline & \multicolumn{2}{|c|}{ Ecad- Membrane } & \multicolumn{2}{|c|}{ Ecad- Plasm } & \multicolumn{2}{|c|}{ Vimentin } \\
\hline & - & + & - & + & - & + \\
\hline \multicolumn{7}{|l|}{ Gender } \\
\hline Male & 18 & 23 & 12 & 29 & 25 & 16 \\
\hline Female & 10 & 14 & 9 & 15 & 13 & 11 \\
\hline \multicolumn{7}{|l|}{ Age } \\
\hline$\leq 40$ & 7 & 6 & 5 & 8 & 7 & 6 \\
\hline$>40$ & 21 & 31 & 16 & 36 & 31 & 21 \\
\hline \multicolumn{7}{|c|}{ Differentiation } \\
\hline Well & 6 & 6 & 4 & 8 & 10 & 2 \\
\hline Mediate & 20 & 30 & 15 & 35 & 28 & 22 \\
\hline Poor & 2 & 1 & 2 & 1 & & 3 \\
\hline \multicolumn{7}{|c|}{ Lymphoid metastasis } \\
\hline No & 18 & 30 & 16 & 32 & 32 & 16 \\
\hline Yes & 10 & 7 & 5 & 12 & 6 & 11 \\
\hline \multicolumn{7}{|l|}{ Stage } \\
\hline I - II & 13 & 29 & 15 & 27 & 29 & 13 \\
\hline I - II & 15 & 8 & 6 & 17 & 9 & 14 \\
\hline \multicolumn{7}{|c|}{ Survival time (months) } \\
\hline & $19.79 \pm 18.27$ & $58.19 \pm 30.37$ & $51.38 \pm 35.45$ & $37.00 \pm 29.59$ & $53.63 \pm 31.68$ & $24.78 \pm 24.36$ \\
\hline
\end{tabular}

Table 2. Relation of E-cadherin with vimentin in TSCC.

\begin{tabular}{|l|c|c|c|c|}
\hline & \multicolumn{2}{|c|}{ Ecad-Membrane } & \multicolumn{2}{c|}{ Ecad-Plasm } \\
\hline & - & + & - & + \\
\hline Vimentin & 7 & 31 & 13 & 25 \\
\hline- & 21 & 6 & 8 & 19 \\
\hline
\end{tabular}

Table 3. Relation of E-cadherin and vimentin with MACC1 in TSCC.

\begin{tabular}{|l|c|c|c|c|c|c|}
\hline & \multicolumn{2}{|c|}{ Ecad- Membrane } & \multicolumn{2}{c|}{ Ecad- Plasm } & \multicolumn{2}{c|}{ Vimentin } \\
\hline & - & + & - & + & - & + \\
\hline MACC1 & 9 & 30 & 13 & 26 & 30 & 9 \\
\hline Low & 9 & 7 & 8 & 18 & 8 & 18 \\
\hline High & 19 & 7 & & & & \\
\hline
\end{tabular}

correlated with MACC1 expression in TSCC $(r=0.459, \mathrm{P}=0.000)$. No association was found between the E-cadherin cytoplasmic expression and MACC1 $(\mathrm{P}=0.830)$, as showed in Table 3.

Figure 1, The expression of E-cadherin (A-D), and vimentin(E-G) in normal squamous epithelium and tumors. E-Cadherin is expressed most in cytomembranes and occasional in cytoplasm of normal cells and $\mathrm{AH}(\mathrm{A})$ and tumor cells(B-D), with obvious staining in cytoplasm of lower differentiated tumors(B) and in cytomembranes of well differentiated tumors(C). Weak staining is showed in both cytomenbrane and cytoplasm in the same tumor(D), which suggests that reduction of membranous expression may not result from protein transport barriers. Normal squamous epithelium and $\mathrm{AH}$ are negative for vimentin(E). Vimentin is expressed obviously in cytoplasm of poor-differentiated tumor islands $(F)$ and the periphery of proliferative islands in the cutinized tumor $(\mathrm{G})$. MACC1 is expressed in the cytoplasm of tumor cells $(\mathrm{H})$.

Figure 2 A, Kaplan-Meier estimates of the overall survival time of patients with E-cadherin membranous positive expression vs. patients with negative expression. The patients with positive E-cadherin membranous expression demonstrated a significantly longer overall survival time $(\mathrm{P}=0.000)$. B. Kaplan-Meier estimates of the overall survival time of patients with E-cadherin cytoplasmic positive expression $v$ s. patients with negative expression. No difference of overall survival time was found between those two groups $(\mathrm{P}=0.117)$. C. Kaplan-Meier estimates of the overall survival time of patients with vimentin positive expression vs. patients with negative expression. The patients with positive vimentin expression demonstrated a significantly shorter overall survival time $(\mathrm{P}=0.043)$.

\section{Discussion}

E-cadherin is an important cell adhesion molecule and signal transduction factor in epithelial tissues, and can prevent and reduce tumor cell adhesion. Immunohistochemically, E-cadherin staining is localized in cell membrane or mainly in cell membrane $[4,12]$. In the current study, E-cadherin expression localized mainly in cell membrane and occasionally in cytoplasm at the ANSE. Reduction of E-cadherin expression in cell membrane and abnormal expression in cytoplasm was found both in the $\mathrm{AH}$ and tumors. As a calcium-dependent transmembrane glycoprotein, E-cadherin immunoexpression in cytoplasm may not have the normal function of cell adhesion. This manifestation is reflective of an early and preinvasive event in the neoplastic transformation of oral squamous epithelia during which E-cadherin accumulates in the cell cytoplasm. Dysfunction immunoexpression of E-cadherin is involved in carcinogenesis and malignancy. The similar change of E-cadherin expression was demonstrated in the squamous epithelium in all nonneoplastic vulvar lesions and vulvar intraepithelial neoplasm [13]. Vimentin is a mesenchymal marker that can be acquired during EMT but its expression is low and most difficult to be quantified, as compared with other EMT markers that indicate a mesenchymal-like phenotype [8]. In the current study, no vimentin immunoexpression was found in either the ANSE or the AH. Though both E-cadherin and vimentin are EMT markers, expression of vimentin is too low to detect immunohistochemically or not present in precancerous lesion of oral squamous carcinoma.

E-cadherin expresses both membranous and cytoplasmic, with the membrane pattern decreasing simultaneously with the decrease of the differentiation degree, while the cytoplasmic pattern has an opposite behavior [14]. Several studies also demonstrated that reduced immunoexpression of this marker in oral squamous cell carcinoma correlates with higher stage and grade of disease, lymph 
node metastasis, tumor invasive behavior and carcinogenesis, which suggests that those patients have a poor prognosis [15-18]. In current study, only $37(56.9 \%)$ cases were recorded as positive for E-cadherin membranous expression although we noticed E-cadherin expression in all the investigated cases. Reduction of E-cadherin membranous expression was significantly associated with more advanced stage, only $8(34.8 \%)$ cases were positive in T3/4 while $29(69.0 \%)$ in T1/2. The survival time was $(19.79 \pm 18.27)$ months in the patients of negative group, which was much less than that of $(58.19 \pm 30.37)$ months in the patients of positive group. However, the increase of E-cadherin cytoplasmic expression was not associated with clinocopathological parameters (tumor differentiation, lymphoid metastasis, TNM stage, and survival time). No relationship was found between the reduction of membranous E-cadherin expression and the increase of cytoplasmic E-cadherin expression, which suggested that reduction of membranous expression might not result from protein transport barriers. Down regulation of E-cadherin has been known as the key step for the invasive phase of carcinoma, and its dominant transcriptional repression has been reported to be largely responsible for the loss of E-cadherin expression [19,20].

Vimentin is an important EMT marker. Cells expressing vimentin have a higher mobility, being capable of invasion and proliferation, and also have tumorigenic potential [21]. An increased vimentin expression is associated with local recurrence, invasiveness and metastasis [22-24]. In current study, 27 (40\%) of these 65 TSCC cases had more than $10 \%$ immunoreactive tumor cells to this marker. The reactivity was more obvious in tumor cells from both the centre of poor-differentiated tumor islands and the periphery of proliferative islands in the cutinized tumor. However, the increase of vimentin expression was not associated with tumor differentiation. Increase of vimentin expression was significantly associated with lymphoid metastasis of TSCC. 11 (64.7\%) cases were positive for vimentin in the group with lymphoid metastasis and $16(33.3 \%)$ positive for vimentin in the group without lymphoid metastasis. Increase of vimentin expression was significantly associated with more advanced stage, only 13 (31.0\%) cases were positive in stage I and II while $29(60.9 \%)$ in stage III and IV. The survival time was $(53.63 \pm 31.69)$ months in the patients of negative group, which was much longer than that of $(24.78 \pm 24.36)$ months in the patients of positive group. Investigation of E-cadherin/vimentin co expression showed that there was a direct correlation between the reductions of E-cadherin membranous expression with an increase of vimentin reactivity.

MACC1 is a newly identified oncogene, and our previous study has revealed that MACC1 influences the prognosis of TSCC patients [6]. MACC1 overexpression upregulates EMT factor and induces changes to the markers of EMT including E-cadherin and wimentin in gastric carcinoma [7]. This study revealed that the high expression of MACC1 in TSCC was associated with the reduction of E-cadherin membranous expression and increase of vimentin expression. However, no association was found between MACC1 and the E-cadherin cytoplasmic expression. MACC1 promotes gastric cancer cell proliferation and invasion by inducing EMT through activation of the hepatocyte growth factor (HGF)/c-Met signaling pathway [7]. HGF induces cell migration in oral squamous cell carcinomas [25]. We suppose that MACC1 promotes EMT through activation of the HGF/cMet signaling pathway in TSCC.

Accounting that the reduction of E-cadherin membranous expression was associated with advanced stage and less survival time, with expression of vimentin, with expression of MMAC1, it might be more important than increase of E-cadherin cytoplasmic expression in TSCC.

\section{Conclusion}

Investigating the E-cadherin and vimentin in TSCC, we found that abnormal expression of E-cadherin and vimentin in tumor tissues suggested that the EMT process might occur and play an important role in the development of TSCC. Abnormal expression of E-cadherin included reduction of membranous expression and increase of cytoplasmic expression, and the reduction of membranous expression might be more important. The abnormal expression of E-cadherin and vimentin was associated with MACC1 in TSCC.

\section{Acknowledgement}

The present study was supported by grants from Health Development Planning Commission of Guangdong Province (No. A2014622) and the Fundamental Research Funds for the Central Universities (\#13ykpy26).

\section{References}

1. Amar A, Rapoport A, Curioni OA, Dedivitis RA, Cernea CR, Brandão LG (2013) Prognostic value of regional metastasis in squamous cell carcinoma of the tongue and floor of mouth. Braz J Otorhinolaryngol.79: 734-737. [Crossref]

2. Huang Z, Huang H, Li H, Chen W, Pan C (2009) EMMPRIN expression in tongue squamous cell carcinoma. J Oral Pathol Med 38: 518-523. [Crossref]

3. Torre LA, Bray F, Siegel RL, Ferlay J, Lortet-Tieulent J, et al. (2015) Global cancer statistics, 2012. CA Cancer J Clin 65: 87-108. [Crossref]

4. Gurzu S, Turdean S, Kovecsi A, et al (2015) Epithelial-mesenchymal, mesenchymalepithelial, and endothelial-mesenchymal transitions in malignant tumors: An update. World J Clin Cases. 3: 393-404. [Crossref]

5. Stein U, Burock S, Herrmann P, Wendler I, Niederstrasser M, et al. (2012) Circulating MACC1 transcripts in colorectal cancer patient plasma predict metastasis and prognosis. PLoS One 7: e49249. [Crossref]

6. Li HF, Liu YQ, Shen ZJ, Gan XF, Han JJ et al. (2015) Downregulation of MACC1 inhibits invasion, migration and proliferation, attenuates cisplatin resistance and induces apoptosis in tongue squamous cell carcinoma. Oncol Rep. 33: 651-660. [Crossref]

7. Wang L, Wu Y, Lin L, Liu P, Huang H et al. (2013) Metastasis-associated in colon cancer-1 upregulation predicts a poor prognosis of gastric cancer, and promotes tumor cell proliferation and invasion. Int J Cancer. 133: 1419-1430. [Crossref]

8. Liu F, Zhou Y, Zhou D, Kan M, Niu X, et al. (2014) Whole DNA methylome profiling in lung cancer cells before and after epithelial-to-mesenchymal transition. Diagn Pathol 9: 66. [Crossref]

9. Mahomed F, Altini M, Meer S (2007) Altered E-cadherin/beta-catenin expression in oral squamous carcinoma with and without nodal metastasis. Oral Dis 13: 386-392. [Crossref]

10. Wang C, Huang H, Huang Z, Wang A, Chen X, et al. (2011) Tumor budding correlates with poor prognosis and epithelial-mesenchymal transition in tongue squamous cell carcinoma. J Oral Pathol Med 40: 545-551. [Crossref]

11. Guo T, Yang J, Yao J, Zhang Y, Da M, et al. (2013) Expression of MACC1 and c-Met in human gastric cancer and its clinical significance. Cancer Cell Int 13: 121. [Crossref]

12. Zhou J, Tao D, Xu Q, Gao Z, Tang D (2015) Expression of E-cadherin and vimentin in oral squamous cell carcinoma. Int J Clin Exp Pathol 8: 3150-3154. [Crossref]

13. Li B, Zhang Q, Ouyang L, Jia L, Han X, et al (2013) Aberrant staining patterns of E-cadherin and $\beta$-catenin: a potential diagnostic value for distinguishing vulvar intraepithelial neoplasia from non-neoplastic vulvar lesions. Int J Clin Exp Pathol. 6: 1362-1366. [Crossref]

14. Afrem MC, Mărgăritescu C, Crăiţoiu MM, Ciucă M, Şarlă CG, Cotoi OS (2014) The immunohistochemical investigations of cadherin "switch" during epithelialmesenchymal transition of tongue squamous cell carcinoma. Rom J Morphol Embryol. 55(3 Suppl): 1049-1056. [Crossref]

15. Wang C, Liu X, Chen Z, Huang H, Jin Y, et al. (2013) Polycomb group protein EZH2mediated E-cadherin repression promotes metastasis of oral tongue squamous cell 
carcinoma. Mol Carcinog 52: 229-236. [Crossref]

16. Huber GF, Züllig L, Soltermann A, Roessle M, Graf N, et al. (2011) Down regulation of E-Cadherin (ECAD) - a predictor for occult metastatic disease in sentinel node biopsy of early squamous cell carcinomas of the oral cavity and oropharynx. $B M C$ Cancer 11: 217:1-8. [Crossref]

17. Vered M, Allon I, Buchner A, Dayan D (2012) E-cadherin in oral SCC: an analysis of the confusing literature and new insights related to its immunohistochemical expression. Histol Histopathol. 27: 141-150. [Crossref]

18. Sakamoto K, Imanishi Y, Tomita T, Shimoda M, Kameyama K, et al. (2012) Overexpression of SIP1 and downregulation of E-cadherin predict delayed neck metastasis in stage I/II oral tongue squamous cell carcinoma after partial glossectomy. Ann Surg Oncol 19: 612-619. [Crossref]

19. Zhou BP, Deng J, Xia W, Xu J, Li YM, et al. (2004) Dual regulation of Snail by GSK3beta-mediated phosphorylation in control of epithelial-mesenchymal transition. $\mathrm{Nat}$ Cell Biol 6: 931-940. [Crossref]

20. Birchmeier W, Behrens J, Weidner KM, Frixen UH, Schipper J (1991) Dominant and recessive genes involved in tumor cell invasion. Curr Opin Cell Biol 3: 832-840. [Crossref]
21. Paccione RJ, Miyazaki H, Patel V, Waseem A, Gutkind JS, et al. (2008) Keratin downregulation in vimentin-positive cancer cells is reversible by vimentin RNA interference, which inhibits growth and motility. Mol Cancer Ther 7: 2894-2903. [Crossref]

22. Liu LK, Jiang XY, Zhou XX, Wang DM, Song XL, Jiang HB (2010) Upregulation of vimentin and aberrant expression of Ecadherin/beta-catenin complex in oral squamous cell carcinomas: correlation with the clinicopathological features and patient outcome. Mod Pathol. 23: 213-224. [Crossref]

23. Mandal M, Myers JN, Lippman SM, Johnson FM, Williams MD, et al. (2008) Epithelial to mesenchymal transition in head and neck squamous carcinoma: association of $\mathrm{Src}$ activation with E-cadherin down-regulation, vimentin expression, and aggressive tumor features. Cancer. 112: 2088-2100. [Crossref]

24. Nijkamp MM, Span PN, Hoogsteen IJ, van der Kogel AJ, Kaanders JH, et al. (2011) Expression of E-cadherin and vimentin correlates with metastasis formation in head and neck squamous cell carcinoma patients. Radiother Oncol 99: 344-348. [Crossref]

25. Brusevold IJ, Aasrum M, Bryne M, Christoffersen T (2012) Migration induced by epidermal and hepatocyte growth factors in oral squamous carcinoma cells in vitro: role of MEK/ERK, p38 and PI-3 kinase/Akt. J Oral Pathol Med 41: 547-558. [Crossref]

Copyright: (C2016 Wu G. This is an open-access article distributed under the terms of the Creative Commons Attribution License, which permits unrestricted use, distribution, and reproduction in any medium, provided the original author and source are credited. 